\title{
Optica
}

\section{Temporal, spectral, and polarization dependence of the nonlinear optical response of carbon disulfide: supplementary material}

\author{
Matthew Reichert ${ }^{1}$, Honghua Hu ${ }^{1}$, Manuel R. Ferdinandus ${ }^{1,2}$, Marcus \\ Seidel $^{1}$, Peng ZhaO ${ }^{1}$, Trenton R. Ensley ${ }^{1}$, Davorin PeCeli ${ }^{1}$, Jennifer M. \\ ReED $^{1}$, DMItry A. FISHMAN ${ }^{1}$, SCOTt WebSTER ${ }^{1}$, DAVID J. HAGAN ${ }^{1,3}$, AND ERIC \\ W. VAN STRYLAND ${ }^{1,3, *}$
}

${ }^{1}$ CREOL, The College of Optics and Photonics, University of Central Florida, Orlando, FL, 32816, USA

${ }^{2}$ Department of Engineering Physics, Air Force Institute of Technology, Dayton, $\mathrm{OH} 45433$

${ }^{3}$ Department of Physics, University of Central Florida, Orlando, FL, 32816, USA

*Corresponding author: ewvs@creol.ucf.edu

Published 18 December 2014

This document provides supplementary information to "Temporal, spectral, and polarization dependence of the nonlinear optical response of carbon disulfide," http://dx.doi.org/10.1364/optica.1.000436. A derivation of the Eq. (9) from the wave equation is provided as well as an example of typical Z-scan data. (C) 2014 Optical Society of America http://dx.doi.org/10.1364/optica.1.000436.s001

\section{DERIVATION OF EQUATION (9)}

We begin with the nonlinear wave equation [1],

$$
\nabla^{2} \mathbf{E}-\frac{n_{0}^{2}(\omega)}{c^{2}} \frac{\partial^{2} \mathbf{E}}{\partial t^{2}}=\mu_{0} \frac{\partial^{2} \mathbf{P}_{N L}}{\partial t^{2}}
$$

where $\mathbf{E}$ is the electric field, $n_{0}$ is the real linear refractive index (neglecting linear loss), $c$ is the speed of light, $\mu_{0}$ is the vacuum permeability, and $\mathbf{P}_{N L}$ is the nonlinear polarization. We are interested in the evolution of the probe field, which we write as $\mathbf{E}=1 / 2 \mathcal{E} e^{i(k z-\omega t)} \hat{\mathbf{e}}+c . c$ In the thin sample approximation, the electric field does not change in the transverse directions, hence $\nabla^{2} \mathbf{E} \approx \partial^{2} \mathbf{E} / \partial z^{2}$. The left hand side of Eq. (S1), applying the slowly varying envelope approximation (SVEA) [1] $\left(\left|\partial^{2} \varepsilon / \partial z^{2}\right| \ll\right.$ $|2 k \partial \mathcal{E} / \partial z|$ and $\left.\left|\partial^{2} \varepsilon / \partial t^{2}\right| \ll|2 \omega \partial \mathcal{E} / \partial t|\right)$, using $k=n_{0} \omega / c$, and dropping the complex conjugate, is then

$$
i k\left(\frac{\partial}{\partial z}+\frac{1}{v} \frac{\partial}{\partial t}\right) \varepsilon e^{i(k z-\omega t)},
$$

where we have expanded $n_{0}(\omega)$ in a Taylor series, $n_{0}(\omega) \approx n_{0}+$ $\omega \partial n_{0} / \partial \omega \equiv n_{g}$, and $v=c / n_{g}$ is the group velocity. The polarization that propagates in the direction of the probe field at the same frequency may be written as $\mathbf{P}_{N L}=1 / 2 \mathcal{P}_{N L} e^{i(k z-\omega t)} \widehat{\mathbf{p}}+$ c.c. , where $\mathcal{P}_{N L}$ is the third-order polarization amplitude, and $\widehat{\mathbf{p}}$ is the nonlinear polarization unit vector. The right hand side of Eq. (S1), applying the SVEA $\left(\left|\partial^{2} \mathcal{P}_{\mathrm{NL}} / \partial t^{2}\right| \ll\left|2 \omega \partial \mathcal{P}_{\mathrm{NL}} / \partial t\right| \ll\left|\omega^{2} \mathcal{P}_{\mathrm{NL}}\right|\right)$, and dropping the complex conjugate, is then

$$
-\frac{\mu_{0} \omega^{2}}{2} \mathcal{P}_{N L} e^{i(k z-\omega t)} .
$$

For frequencies far from electronic and nuclear resonances, the Born-Oppenheimer approximation may be applied. It allows the separation of the bound-electronic and nuclear contributions to the nonlinear polarization, which may be written as $\mathcal{P}_{N L}=\mathcal{P}_{e l}+$ $\mathcal{P}_{n u}$ [2]. To third-order, the bound-electronic contribution is given by

$$
\mathbf{P}_{e l, i}^{(3)}=\varepsilon_{0} \chi_{e l, i j k l}^{(3)} \mathbf{E}_{j} \mathbf{E}_{k} \mathbf{E}_{l},
$$

where $\chi_{e l}^{(3)}$ is the real bound-electronic third-order susceptibility, and a summation over subscripts $j, k$, and $l$ is assumed. The total electric field is a sum of the probe and excitation fields. Keeping only the products of fields that oscillate as $e^{i(k z-\omega t)}$, and assuming the probe field is much weaker than the excitation, $\mathcal{P}_{\text {el }}^{(3)}$ is given by

$$
\mathcal{P}_{e l}^{(3)}=3 \frac{\varepsilon_{0}}{2} \chi_{e l}^{(3)}\left(\omega ; \omega, \omega_{e},-\omega_{e}\right) \mathcal{E}\left|\mathcal{E}_{e}\right|^{2},
$$

where the subscript $e$ indicates the excitation, intrinsic permutation symmetry has been used [1]. 
The nuclear contribution of the nonlinear polarization is given by [2]

$$
\begin{array}{r}
\mathbf{P}_{n u}^{(3)}(t)=\varepsilon_{0} \iint_{-\infty}^{\infty} \int_{n u}^{(3)}\left(t-t^{\prime}, t-t^{\prime \prime}, t-t^{\prime \prime \prime}\right) \\
\cdot \mathbf{E}\left(t^{\prime}\right) \mathbf{E}\left(t^{\prime \prime}\right) \mathbf{E}\left(t^{\prime \prime \prime}\right) d t^{\prime} d t^{\prime \prime} d t^{\prime \prime \prime} .
\end{array}
$$

For nonresonant responses, $\chi_{n u}^{(3)}\left(t-t^{\prime}, t-t^{\prime \prime}, t-t^{\prime \prime \prime}\right)=$ $\chi_{n u}^{(3)}\left(t-t^{\prime}\right) \delta\left(t-t^{\prime \prime}\right) \delta\left(t-t^{\prime \prime \prime}\right)$ [2], and thus where $\delta(t)$ is the Dirac delta function. Thus, Eq. (S6) simplifies to

$$
\mathbf{P}_{n u}^{(3)}(t)=\varepsilon_{0} \mathbf{E}(t) \int_{-\infty}^{\infty} \chi_{n u}^{(3)}\left(t-t^{\prime}\right) \mathbf{E}\left(t^{\prime}\right) \mathbf{E}\left(t^{\prime}\right) d t^{\prime},
$$

where $\chi_{n u}^{(3)}$ is the (non-instantaneous) nuclear component of the third-order susceptibility. Because we have assumed the frequencies are far from nuclear resonances, the only terms of product of two fields in the integrand that survive integration are those that are complex conjugates of one another, e.g $e^{i \omega_{e} t} e^{-i \omega_{e} t}=1$. In addition, we assume the nondegenerate case $\omega \neq \omega_{e}$, and that the probe is weak. Substituting the expression for the electric fields and nonlinear polarization yields

$$
\mathcal{P}_{n u}^{(3)}(t)=\frac{\varepsilon_{0}}{2} \mathcal{E}(t) \int_{-\infty}^{\infty} \chi_{n u}^{(3)}\left(t-t^{\prime}\right)\left|\mathcal{E}_{e}\left(t^{\prime}\right)\right|^{2} d t^{\prime} .
$$

Eq. (S3) now given by

$$
\begin{aligned}
& -\frac{k_{0}^{2}}{4} \varepsilon e^{i(k z-\omega t)} \\
& \cdot\left(3 \chi_{e l}^{(3)}\left|\mathcal{E}_{e}\right|^{2}+\int_{-\infty}^{\infty} \chi_{n u}^{(3)}\left(t-t^{\prime}\right)\left|\mathcal{E}_{e}\left(t^{\prime}\right)\right|^{2} d t^{\prime}\right),
\end{aligned}
$$

where $\varepsilon_{0} \mu_{0} \omega^{2}=k_{0}^{2}$. We may now write the magnitude of the excitation field in terms of its irradiance $\left|\varepsilon_{e}\right|^{2}=2 I_{e} /\left(n_{0, e} \varepsilon_{0} c\right)$, where the nonlinear wave equation becomes

$$
\begin{aligned}
& i\left(\frac{\partial}{\partial z}+\frac{1}{v} \frac{\partial}{\partial t}\right) \mathcal{E}=-k_{0} \mathcal{E} \\
& \left(2 \frac{3 \chi_{e l}^{(3)}}{4 n_{0} n_{0, e} \varepsilon_{0} c} I_{e}+\int_{-\infty}^{\infty} \frac{2 \chi_{n u}^{(3)}\left(t-t^{\prime}\right)}{4 n_{0} n_{0, e} \varepsilon_{0} c} I_{e}\left(t^{\prime}\right) d t^{\prime}\right) .
\end{aligned}
$$

The nonlinear refractive index is given by

$$
n_{2, e l}=\frac{3 \chi_{e l}^{(3)}}{4 n_{0} n_{0, e} \varepsilon_{0} c}
$$

and

$$
R(t)=\frac{2 \chi_{n u}^{(3)}(t)}{4 n_{0} n_{0, e} \varepsilon_{0} c} .
$$

Substituting Eqs. (S11) and (S12) into (S10) gives

$i\left(\frac{\partial}{\partial z}+\frac{1}{v} \frac{\partial}{\partial t}\right) \varepsilon+$

$k_{0} \mathcal{E}\left(2 n_{2, e l} I_{e}(t)+\int_{-\infty}^{\infty} R\left(t-t^{\prime}\right) I_{e}\left(t^{\prime}\right) \mathrm{d} t^{\prime}\right)=0$,

\section{Z-scan Data}

Fig. S1(a) shows an example of Z-scan measurements including open aperture measurements ZnSe (of $511 \mu \mathrm{m}$ thick) for spot size and pulse width determination. The pulse energy was $1.5 \mathrm{~nJ}$, spot size of $19.5 \mu \mathrm{m}\left(\mathrm{HW} 1 / \mathrm{e}^{2} \mathrm{M}\right)$, and pulse width of $180 \mathrm{fs}$ (FWHM). The curve corresponds to a fit with $\alpha_{2}=5.6 \times 10^{-11} \mathrm{~m} / \mathrm{W}$. Fig. S1(b) shows a typical closed aperture Z-scan measurement a $1 \mathrm{~mm}$ cuvette of $\mathrm{CS}_{2}$ for the same parameters, but at several input energies. The close aperture linear transmission $S=0.33$. The fits correspond to $n_{2, \text { eff }}=6.3 \times 10^{-19} \mathrm{~m}^{2} / \mathrm{W}$.
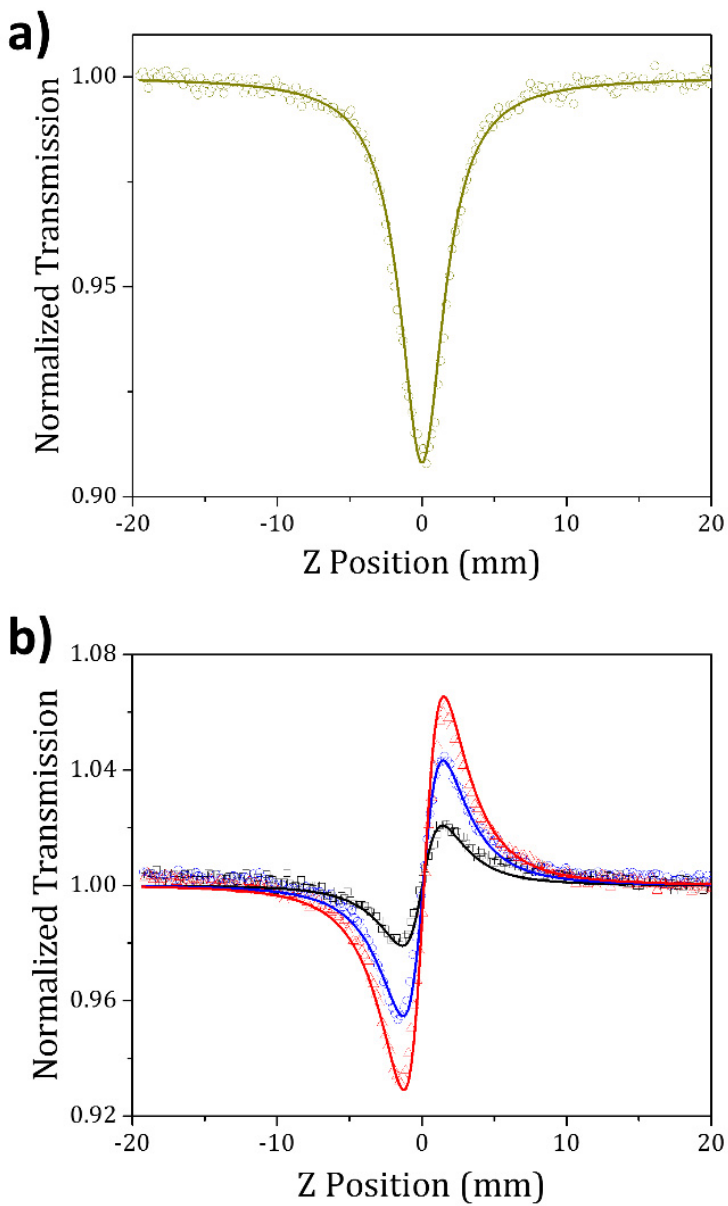

Fig. S1. Typical a) open aperture data (points) and fits (curves) Z-scan of $\mathrm{ZnSe}$, and b) closed aperture Z-scan of $\mathrm{CS}_{2}$ taken at multiple energies of $3.5 \mathrm{~nJ}$ (black squares), $7.4 \mathrm{~nJ}$ (blue circles), and $11 \mathrm{~nJ}$ (red triangles).

\section{References}

1. R. W. Boyd, "Nonlinear Optics," $3^{\text {rd }}$ ed. ISBN 978-0-12-369470-6. Published by Academic Press/Elsevier, Inc. Oxford, UK, 2008. 1(2008).

2. R. W. Hellwarth, "Third-order susceptibilities of liquids and solids," in Progress in Quantum Electronics, J. H. Sanders, and S. Stenholm, eds. Vol. 5, (Pergamon Press, 1977), pp. 1-68

which is Eq. (9). 